\title{
Epidemiology of Schistosomiasis and Soil-Transmitted Helminth Coinfections among Schoolchildren Living in Lambaréné, Gabon
}

\author{
Jean Claude Dejon-Agobé, ${ }^{1,2}$ Yabo Josiane Honkpehedji, ${ }^{1,3}$ Jeannot Fréjus Zinsou, ${ }^{1,3}$ Jean Ronald Edoa, ${ }^{1}$ \\ Bayodé Roméo Adégbitè, ${ }^{1}$ Ance Mangaboula, ${ }^{1}$ Selidji Todagbe Agnandji, ${ }^{1,4}$ Ghyslain Mombo-Ngoma, ${ }^{1,4,5}$ Michael Ramharter, ${ }^{1,5}$ \\ Peter Gottfried Kremsner, ${ }^{1,4,6,7}$ Bertrand Lell, ${ }^{1,8}$ Martin Peter Grobusch, , ${ }^{1,2}$ and Ayôla Akim Adegnika ${ }^{1,3,4,6,7 *}$ \\ ${ }^{1}$ Centre de Recherches Médicales de Lambaréné (CERMEL), Lambaréné, Gabon; ${ }^{2}$ Division of Internal Medicine, Department of Infectious \\ Diseases, Center of Tropical Medicine and Travel Medicine, Amsterdam University Medical Centers, location AMC, University of Amsterdam, \\ Amsterdam, The Netherlands; ${ }^{3}$ Department of Parasitology, Leiden University Medical Center, Leiden, The Netherlands; ${ }^{4}$ Institut für \\ Tropenmedizin, Eberhad Karls Universität Tübingen, Tübingen, Germany; ${ }^{5}$. Department of Medicine, Bernhard Nocht Institute for Tropical \\ Medicine, University Medical Centre-Hamburg-Eppendorf, Hamburg, Germany; ${ }^{6}$ German Center for Infection Research (DZIF), African Partner \\ Institution, CERMEL, Lambaréné, Gabon; ${ }^{7}$ German Center for Infection Research (DZIF), Partner Site, Tübingen, Germany; ${ }^{8}$ Division of Infectious \\ Diseases and Tropical Medicine, Department of Medicine 1, Medical University of Vienna, Vienna, Austria
}

\begin{abstract}
Schistosomiasis is a parasitic infection highly prevalent in Central Africa where it is co-endemic with many other parasitic infections, including soil-transmitted helminths (STHs). For its optimal control, there is a need of descriptive epidemiological data for each endemic region. The objective of the present study was to determine the epidemiological situation around schistosomiasis in Lambaréné, Gabon. A cross-sectional study was conducted among schoolchildren. One urine sample per day was collected on three consecutive days for the diagnosis of schistosomiasis using a urine filtration technique. One stool sample was collected for the detection of Schistosoma spp. and STH spp. eggs using the Kato-Katz technique, and for larvae, using the coproculture technique. A total of 614 schoolchildren were included in the analysis. The overall prevalence of schistosomiasis and STH infections was 26\% (159/614) and 15\% (70/473), respectively. Human-freshwater contact was the main risk factor for schistosomiasis in the area (relative risk $(R R)=2.96$ [2.20-4.00], $P<0.001)$. Hematuria (RR $=5.53$ [4.30-7.10], $P<0.001)$ and proteinuria $(\mathrm{RR}=2.12[1.63-2.75], P<0.001)$ as well as infection with Trichuris trichiura $(\mathrm{RR}=1.86$ [1.33-2.61], $P=0.002)$ and Ascaris lumbricoides $(\mathrm{RR}=1.96$ [1.19-3.21], $P=0.039$ ) were associated with an increased risk of schistosomiasis. Trichuris trichiura was the highest prevalent STH species in the area. Our study reports a moderate prevalence for schistosomiasis with human-water contact as the main risk factor, whereas the prevalence of STH infections appears to be low. Our results stress the need for the implementation of WHO recommendations for schistosomiasis control.
\end{abstract}

\section{INTRODUCTION}

Schistosomiasis is the most devastating parasitic disease worldwide, second only to malaria. ${ }^{1}$ According to the WHO, an estimated 207 million people may have schistosomiasis in the world. ${ }^{2}$ Of these, more than $90 \%$ live in sub-Saharan Africa (sSA), ${ }^{2}$ which is among the parts of the world where the greatest number of soil-transmitted helminth (STH) infections occurs $^{3}$ and where preschool-age and school-age children are particularly exposed to STHs. Indeed, with a just more than 271 million children exposed to schistosomiasis in 2018, the WHO consider Africa second to Southeast Asia as the part of the world which requires most of the billion preventive chemotherapy for soil-transmitted helminthiases needed globally. ${ }^{4}$ The main species of STHs that infect humans are Ascaris lumbricoides, Trichuris trichiura, and hookworm (Necator americanus and Ancylostoma duodenale).

In SSA, two species of schistosomiasis are predominant: Schistosoma haematobium, causing urogenital schistosomiasis, and Schistosoma mansoni, causing intestinal schistosomiasis. In addition, Schistosoma intercalatum and Strongyloides guineensis, particularly prevalent in Central Africa, are causes of rectal schistosomiasis. For all types of schistosomiasis, the morbidity depends on population factors. As for STH infections, school-age children and young adults seem to bear the brunt of the disease. ${ }^{5}$ In these subpopulations, anemia and malnutrition, among

*Address correspondence to Ayola Akim Adegnika, Centre de Recherches Médicales de Lambaréné (CERMEL), BP: 242, Lambaréné, Gabon. E-mail: aadegnika@cermel.org other consequences, are very often reported to be associated with the disease. ${ }^{6}$

Schistosomiasis involves human contact with infested freshwater. The occurrence of the disease is, therefore, highly related to the environment and population behaviors, and the epidemiology will, thus, widely vary from one region to another. Indeed, absence of safe water, lack of sanitation, and culture-associated behavioral patterns can increase the risk of being infected in some subpopulations. For disease prevention, the WHO recommends a universal approach which includes access to safe water and improvement of sanitation and hygiene education (WASH), in addition to mass drug administration (MDA) with praziquantel (PZQ) and snail control. ${ }^{7}$ Mass drug administration is recommended for areas with moderate or high schistosomiasis prevalence, and the application frequency depends on the prevalence of infection. ${ }^{8}$ This underpins the need to assess the disease prevalence for each particular endemic region.

In Gabon, schistosomiasis cases have been reported already in the $1960 \mathrm{~s} .{ }^{9}$ The disease is present in almost the whole country. ${ }^{10}$ The country is also endemic for STH infections. A national baseline mapping for schistosomiasis and STH infections initiated by the government in 2014 showed a low prevalence of schistosomiasis in the northern and eastern health regions of the country. ${ }^{11}$ For the control of schistosomiasis and STH infections, Gabon has opted for national campaigns of MDA of PZQ and albendazole among schoolchildren. The only known national campaigns of PZQ conducted before the present study which covered Lambaréné town took place in 2016. Lambaréné is the capital of MoyenOgooué, one of the nine provinces of Gabon, located centrally 
in the country and is being known to be endemic for STH infections ${ }^{12}$ and schistosomiasis, with $S$. haematobium as the main Schistosoma species encountered. ${ }^{13,14}$ Whereas epidemiological data are available for STH infections, ${ }^{12}$ little is known about the descriptive epidemiology of schistosomiasis, particularly on the disease's exact prevalence. Understanding the epidemiological situation of schistosomiasis in this semi-urban area and the transmission dynamics over time are of importance, the prevalence of the infection being an indicator of MDA frequency. The objective of the present study was, therefore, to describe the epidemiology of schistosomiasis in Lambaréné, including its coinfection with STHs.

\section{MATERIALS AND METHODS}

Study area. Lambaréné is a semi-urban area with 44,000 inhabitants reported in $2016 .{ }^{15}$ The town is $240 \mathrm{~km}$ distant by road in a southerly direction from Libreville, capital of Gabon, and situated $100 \mathrm{~km}$ south of the equator. The Ogooué River, the main river of the country, is fed by several streams and traverses the town, forming many ponds and lakes, which offer favorable conditions for freshwater snail development. Previous reports have demonstrated the presence of $S$. haematobium in the area. $^{13,14}$ There is some evidence of the presence of intestinal schistosomiasis, too. ${ }^{10,16}$ Despite a municipal water supply network provided by the National Society of Water and Electricity (Société d'Electricité et d'Eau du Gabon), large parts of the population do not have access to safe piped or pump water because of insufficient capacity of the company. Indeed, small streams, tributaries to the Ogooue River, are used more by a part of the local population for water supply, fishing, and household work, and by children mainly for playing. Lambaréné is also known to be endemic for intestinal helminths. ${ }^{12}$

Study population and sampling. A cross-sectional study was conducted from April to July 2016. The target population was primary schoolchildren from Lambaréné. Among the 26 primary schools across Lambaréné, a total of 17 primary schools were asked to participate in the study. These included all governmental primary schools $(n=8)$, all confessional primary schools $(n=6)$, and three randomly selected private primary schools. The schools were distributed across the town. Selection of participants was carried out using a 2-stage cluster sampling procedure. At the first sampling stage, one class batch was selected at random for each academic level (5-year levels) and for each school. For each class selected, all children were invited to participate in the study. The legal representatives were visited at home, and informed consent was requested from them to enable their child to participate in the study. Among those children whose parents granted informed consent, around 10 children were included per class. For the classroom with a size equal to or less than 10 students, all students for whom the parents provided the informed consent were included.

Sample size calculation. A study conducted earlier in ZiléPK villages, a rural area close to Lambaréné, reported a schistosomiasis prevalence of $45 \% .{ }^{17}$ For Lambaréné as a semi-urban area, we hypothesize a one-third decrease in schistosomiasis prevalence, compared with that in the rural Zilé-PK villages setting. Thus, to be able to detect a variation in prevalence of at least 15\%, with $95 \%$ confidence and 0.05 precision using a formula reported elsewhere, ${ }^{18}$ we needed to include at least 323 volunteers in the present study.
Data collection. Study nurses collected demographic data (age, gender, and address) and anthropometric parameters (weight, height, and mid-arm brachial circumference) at school before blood sampling. To inquire about human-water contact and potential treatment received (PZQ and anthelminthic drugs) during the past 6 months, trained field workers applied a standardized questionnaire to participants. For very young children for whom the interviewer judged the veracity of the answer as doubtful, the questions were asked to parents or the primary caretaker at home and the answers weighted. In addition, the interviewers collected schools' and participant houses' global positioning system (GPS) coordinates, using hand-held GPS monitors. All data collected were transcribed into the case report form (CRF) and then digitalized.

Sample collection and laboratory examinations. The research team provided eligible participants with plastic containers and invited them to provide three urine samples on three consecutive days and one stool sample. Samples were collected at school. For each urine sample, urine filtration was performed for the detection of $S$. haematobium eggs using a micro-filter membrane of $12 \mu \mathrm{m}$ (Whatman type) as described elsewhere. ${ }^{19}$ Furthermore, 11 urine parameters, including erythrocyte and protein, were assessed by urine dipstick (Combur 10 Test). We used the Kato-Katz technique for the detection of eggs of STHs in stool samples as described elswhere. ${ }^{20}$ Also, we used the coproculture technique for the detection of hookworm (N. americanus) and S. stercoralis larvae. In detail, approximately $10 \mathrm{mg}$ of sieved stool was collected using a spatula and transferred onto a slide, which was three-time wound with adsorbent tissue and placed inside a petri dish. Twenty milliliters of tap water was then added into the petri dish such that the tissue paper was just soaked and the water did not cover the stool preparation. The petri dish was then placed in an incubator for 7 days with the temperature set at $22-28^{\circ} \mathrm{C}$. Following this incubation period, $10 \mathrm{~mL}$ of liquid obtained from the petri dish by using a syringe was passed through a micro-filter membrane of 10-12 $\mu \mathrm{m}$. The membrane was then transferred onto a glass slide, mounted on a microscope, and read using a low-power objective $(x 10)$ of a light microscope. The slide reading was performed by two independent experienced readers. The result was reported as the number of larvae per $10 \mathrm{mg}$ of stool after calculating the mean larvae count obtained from the pooled results of both readers. In the case of a quantitative (difference $\geq 20 \%$ ) or a qualitative discrepancy between both readers, a third independent reader was required, and the mean of the two closest results was considered as the definitive result.

For all examinations performed, the result was recorded in the CRF and then digitalized. Results of laboratory examinations were reported back to the parents or legal representatives of each participant. Participants found infected were treated with $40 \mathrm{mg} / \mathrm{kg}$ of PZQ once for schistosomiasis and/or $400 \mathrm{mg}$ of albendazole once a day during three consecutive days for STH infection. For other infections when indicated, participants were referred to an appropriate healthcare center.

Statistics. Data were collected and managed using the REDCap electronic data capture too ${ }^{21}$ hosted at Centre de Recherches Médicales de Lambaréné (CERMEL) and exported into R software (v. 3.2.4, R core team, Vienna, Austria) for statistical analysis. Age was used as the categorical variable. The hemoglobin level threshold to define anemia was set as defined by the WHO for sea level. ${ }^{22}$ To determine child 
nutritional status, the BMl-for-age Z-score for children and adolescents aged from 5 to 19 years was applied, as recommended by the $\mathrm{WHO}^{23}$ Intensity of schistosomiasis and STH infections were defined as described elsewhere. ${ }^{24}$ Quantitative and normal distributed variables were summarized by mean and SD. Qualitative data were summarized by prevalence and $95 \% \mathrm{Cl}$. The chi-squared test of independence was performed to assess risk factors and factors associated with schistosomiasis. The level of statistical significance was set as $P<0.05$.

Ethics. The study protocol was approved by CERMEL's scientific committee (SCR-Number: 2016-01) and by the Institutional Ethic Committee of CERMEL (CEI-CERMEL 002/ 2016). Agreement to conduct the study in schools was obtained from the administrative authorities. The study was conducted in line with the Good Clinical Practice principles of the International Conference on Harmonization ${ }^{25}$ and the Declaration of Helsinki. ${ }^{26}$

\section{RESULTS}

Study population. A total of 670 participants consented in writing to participate in the study. Among them, 629 provided urine, stool, and/or blood samples. In the present analysis, we included only those 614 participants with known schistosomiasis status (Figure 1). As depicted in Figure 2A, the study population distribution was homogeneous across the study area. The mean (SD) age of our study population was $10.9 \pm$ 2.7 years, with a 0.95 female-to-male ratio. Normal values of nutritional status were reported for $81 \%$ (95\% Cl: 77.6-84.0) of the study population, whereas $25 \%$ (95\% Cl: $21-28)$ had a hemoglobin level less than $11 \mathrm{~g} / \mathrm{dL}$. Hematuria and proteinuria were present in 21\% (95\% Cl: 18-25) and 20\% (95\% Cl: 16-23) of the study population, respectively, whereas $10 \%$ (95\% Cl: 7-12) presented with both proteinuria and hematuria. Nearly half of the study population (44\% [95\% Cl: 40-48]) had declared having had freshwater contact. Ten per cent $(95 \% \mathrm{Cl}$ : $8-13)$ and $22 \%$ (95\% Cl: 19-26) of our study population have declared having taken PZQ and treatment for STH infection (albendazole) in the last six months before the beginning of the study, respectively (Table 1).

Schistosoma haematobium infection prevalence and intensity. As presented in Table 2, the overall prevalence of schistosomiasis was $26 \%$ (95\% Cl: 23-30). A total of 159 children of 614 who provided urine sample harbored Schistosoma spp. eggs, reflecting a prevalence of $26 \%(95 \% \mathrm{Cl}$ : 22-29) for urogenital schistosomiasis, whereas only three children of 472 who provided a stool sample had Schistosoma spp. eggs in the feces, yielding a prevalence of less than $1 \%$ (95\% Cl: 0-2) for potential intestinal or rectal schistosomiasis. Among the infected children, one harbored eggs in urine and stool concomitantly. For S. haematobium infection, $72 \%$ of 159 infected participants presented with light infection. The geographical distribution of cases of schistosomiasis as shown in Figure 2B reveals that even if schistosomiasis is scattered across the study area, there is some clustering of these cases particularly around the northern entrance of the town which presents a high concentration of cases.

Soil-transmitted helminth infection prevalence and intensity. As shown in Table 2, the overall prevalence of STH infections among the 472 subjects who provided a stool sample was 15\% (95\% Cl: 12-19), with all infections classified

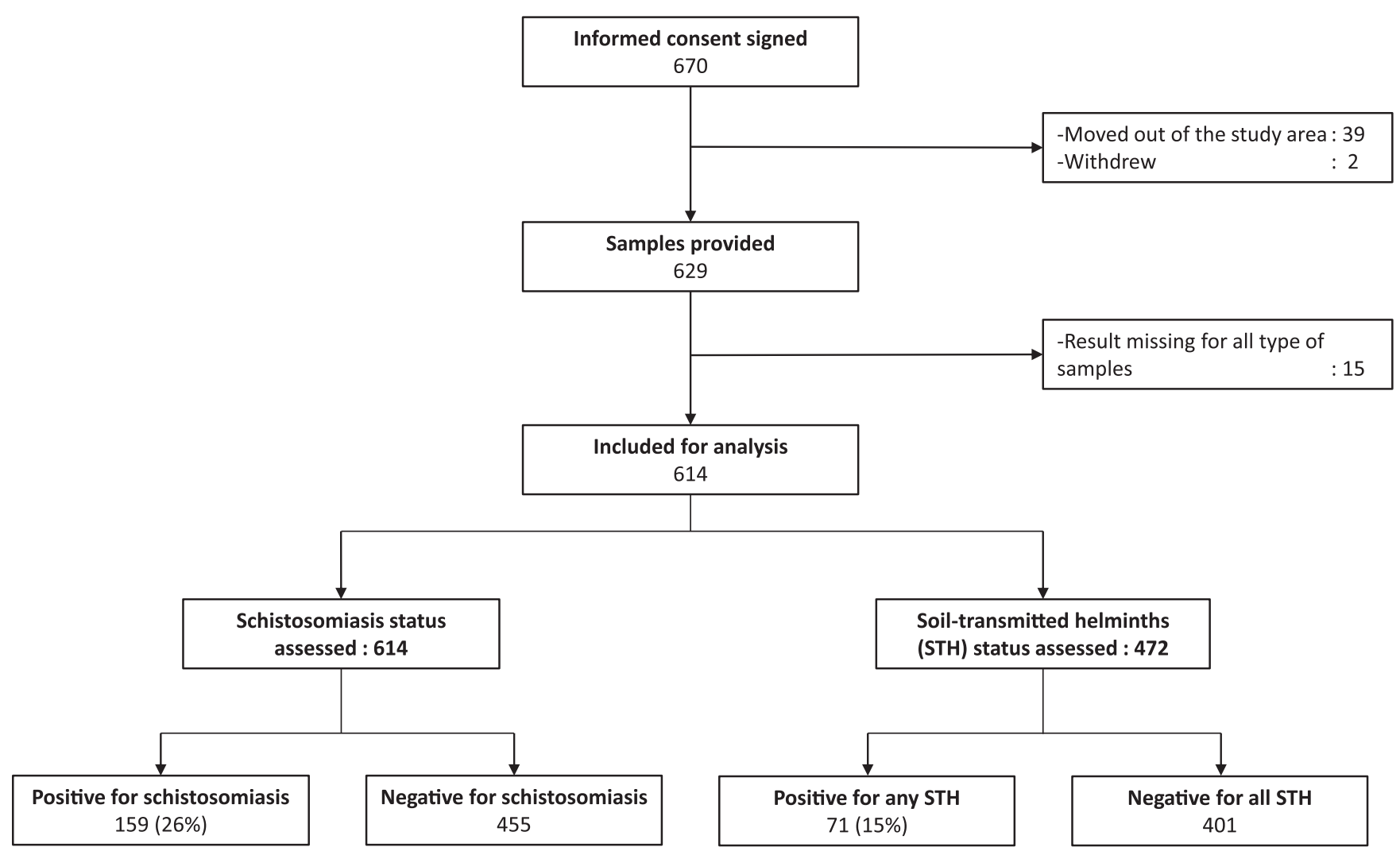

FIGURE 1. Study flowchart representing the number of participants considered at different study time points. 


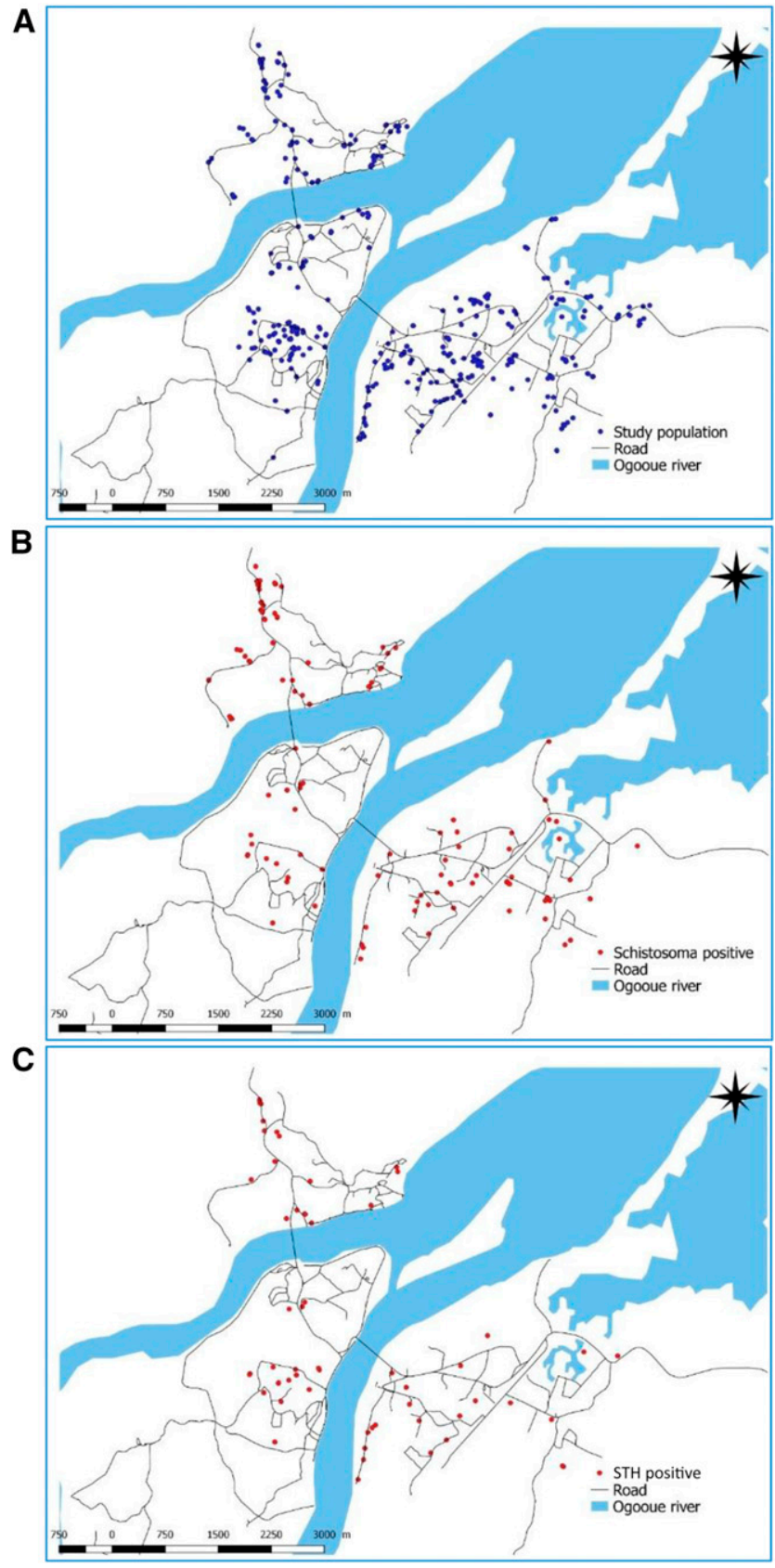

FIGURE 2. Geographical distribution of study participants using QGIS software version 3.2.2 (Free Software Foundation, Boston, MA); (A) overall study population, (B) participants infected with schistosomiasis, and (C) participants infected with soil-transmitted helminths (STH).

as light (Figure 3). The most prevalent STH was T. trichiura, followed by hookworm and $A$. lumbricoides with $10 \%$ (95\% Cl: 8-13), 4\% (95\% Cl: 2-6), and 3\% (95\% Cl: 2-5) prevalence, respectively. Strongyloides stercoralis accounted for $1 \%$ (95\% Cl: 0-3). Coproculture technique was performed to improve the diagnosis of hookworm infection in our study population by detecting the presence of larvae in stool. Among the 18 participants classified as positive for hookworm, six (33\%) were positive for the presence of eggs only in the stool, four $(22 \%)$ were positive for the presence of larvae only in the stool, whereas eight (44\%) were positive for both.
TABLE 1

Characteristics of the 614 study participants, April to July 2016, Lambaréné, Gabon

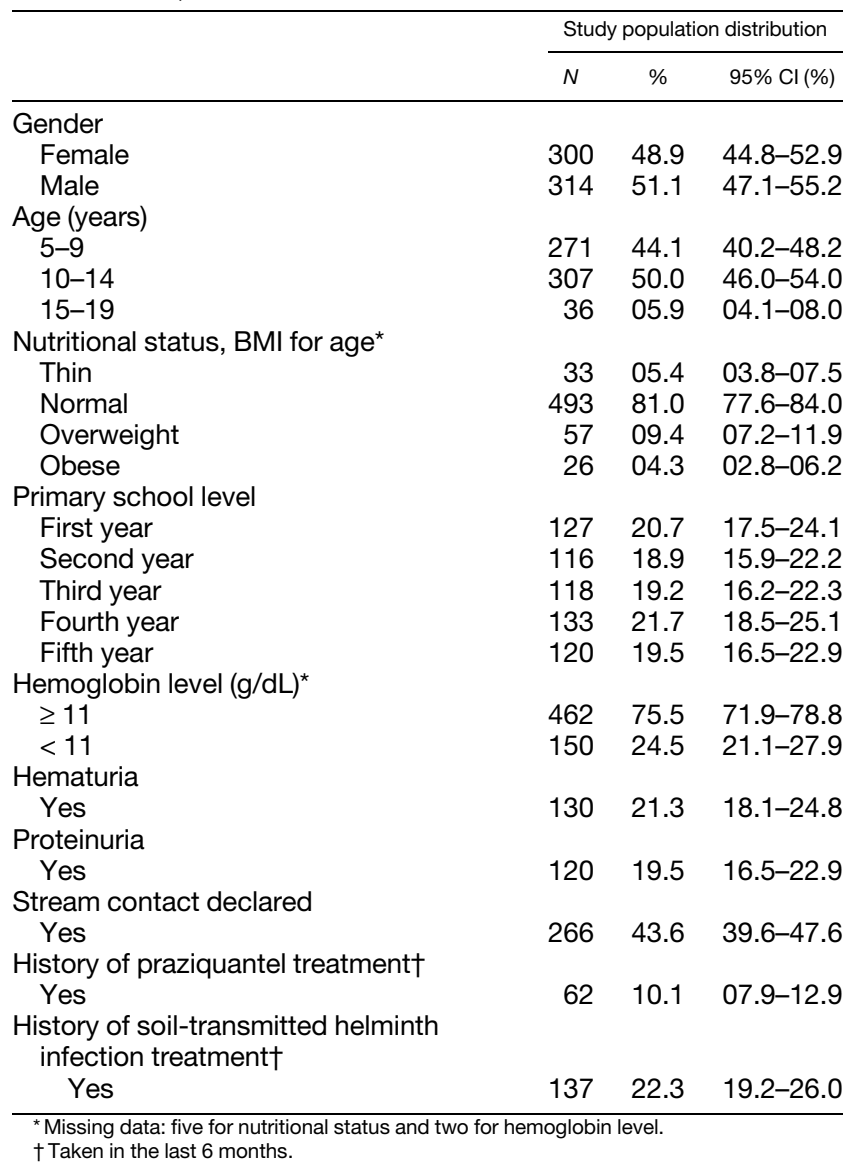

Schistosomiasis and STH coinfections. Assessing concomitant infections with other parasites among the 472 participants who provided stool and urine samples, schistosomiasis was found in coinfection with each STH infection investigated in the study, even for those with very low prevalence. As presented in Table 2, the schistosomiasis coinfection rate with any STH infection was $6 \%(95 \% \mathrm{Cl}: 4-8)$ and the most prevalent coinfection was trichuriasis with 5\% $195 \%$ Cl: 3-7). Regarding coinfections between different species of STHs among the 70 children infected, the prevalence of double infections was $24 \%(95 \% \mathrm{Cl}: 14-34)$, and the two most prevalent pairings of coinfections were trichuriasis-ascariasis (10\% [95\% Cl: 3-17]) and trichuriasis-ancylostomiasis (9\% [95\% Cl: 2-16]). Some cases of triple infections were found, notably ancylostomiasis-strongyloidiasis-ascariasis $(3 \%$ [95\% Cl: 0-7]) and ancylostomiasis-strongyloidiasis-trichuriasis (2\% [95\% Cl: 0-5]).

Factors associated with schistosomiasis. At bivariate analysis as shown in Table 3 , age $(P=0.76)$, gender $(P=0.50)$, and educational level $(P=0.43)$ were not statistically associated with schistosomiasis. On the contrary, freshwater contact was highly statistically associated with schistosomiasis. Children who declared freshwater contact had a thee-time higher risk (RR $=2.96[95 \% \mathrm{Cl}: 2.2-4.0], P<0.001)$ to be infected with $S$. haematobium than those who have not declared a freshwater contact. By contrast, as presented in Table 4, nutritional status $(P=0.61)$ was not associated with 
TABLE 2

Schistosomiasis and STH prevalence and coinfection among the study participants, April to July 2016, Lambaréné, Gabon

\begin{tabular}{|c|c|c|c|c|c|c|}
\hline & \multicolumn{3}{|c|}{ Participants infected } & \multicolumn{3}{|c|}{ Schistosomiasis coinfection } \\
\hline & $n$ & $\%$ & $95 \% \mathrm{Cl}(\%)$ & $n$ & $\%$ & $95 \% \mathrm{Cl}(\%)$ \\
\hline \multicolumn{7}{|l|}{ Schistosoma spp. } \\
\hline Schistosoma haematobium $(N=614)$ & 159 & 25.9 & $22.4-29.5$ & - & - & - \\
\hline$<50$ eggs $/ 10 \mathrm{~mL}$ of urine & 115 & 72.3 & $65.4-79.3$ & - & - & - \\
\hline$\geq 50$ eggs $/ 10 \mathrm{~mL}$ of urine & 44 & 27.7 & 20.7-34.6 & - & - & - \\
\hline Schistosoma intercalatum $(N=472)$ & 3 & 0.6 & $0.1-1.8$ & - & - & - \\
\hline \multicolumn{7}{|l|}{ STH infection $(N=472)$} \\
\hline Trichuris trichiura & 49 & 10.4 & $7.8-13.5$ & 23 & 4.9 & $3.1-7.2$ \\
\hline Hookworm & 18 & 3.8 & $2.3-5.9$ & 4 & 0.8 & $0.2-2.1$ \\
\hline Ascaris lumbricoides & 15 & 3.2 & $1.8-5.2$ & 8 & 1.7 & $0.7-3.3$ \\
\hline Strongyloides stercoralis & 6 & 1.3 & $0.5-2.7$ & 2 & 0.4 & $0.0-1.5$ \\
\hline Any STH & 71 & 15.0 & $11.9-18.6$ & 28 & 5.9 & $4.0-8.5$ \\
\hline
\end{tabular}

schistosomiasis. Taken together, STHs were associated with an increased risk of being infected with schistosomiasis $(\mathrm{RR}=$ 1.52 [95\% Cl: 1.09-2.12], $P=0.02$ ). Considering each species of STH, there was no statistical association for hookworm infection (RR $=0.79[95 \% \mathrm{Cl}: 0.32-1.89], P=0.77)$ and strongyloidiasis ( $\mathrm{RR}=1.19$ [95\% $\mathrm{Cl}: 0.38-3.74], P=0.67)$ with schistosomiasis, but there was a significant increase in risk of being infected with Schistosoma spp. when positive for trichuriasis (RR $=1.82$ [95\% Cl: $1.33-2.56], P=0.003$ ) or for ascariasis (RR $=1.97$ [95\% Cl: $1.20-3.23], P=0.04)$. On the other hand, children with a hemoglobin level less than $11 \mathrm{~g} / \mathrm{dL}$ (RR $=1.54$ [95\% Cl: 1.17-2.02], $P=0.003)$, presenting with hematuria $(\mathrm{RR}=5.53$ [95\% Cl: 4.3-7.1], $P<0.001)$ or proteinuria (RR $=2.12$ [95\% Cl: 1.63-2.75], $P<0.001$ ), had an increasing risk of being positive for schistosomiasis.



FIGURE 3. Egg count distribution for soil-transmitted helminth infections and schistosomiasis after Kato-Katz and urine filtration examinations, respectively, showing ascariasis as the infection presenting with the widest distribution in the number of eggs.

\section{DISCUSSION}

The present study confirms urogenital schistosomiasis in Lambaréné as predominating in the area. We found three participants with stool samples positive for the presence of Schistosoma eggs identified as S. intercalatum eggs, establishing the presence of few cases of intestinal schistosomiasis in the study area. Indeed, if the evidence of $S$. haematobium is widely reported in Lambaréné, ${ }^{12,27}$ only scarce data reveal the presence of $S$. intercalatum ${ }^{10}$ or $S$. guineensis ${ }^{16}$ in the area. Because $S$. haematobium eggs can be found in stool, ${ }^{28}$ and confusion between $S$. intercalatum and $S$. haematobium eggs is possible when the differentiation is carried out based only on their shape and size using a microscopic method, ${ }^{29}$ as we did, it will be relevant to use a molecular method to reconfirm the presence of S. intercalatum and/or S. guineensis in the area.

The main objective of the present study was to determine the prevalence of schistosomiasis in Lambaréné. Our results reveal $26 \%$ prevalence for urogenital schistosomiasis using the urine filtration technique as a diagnostic tool, classifying schistosomiasis prevalence as moderate for Lambaréné's community $^{8}$ and Schistosoma spp. as the most prevalent helminths in this town. To the best of our knowledge, this is the first time the level of schistosomiasis prevalence is assessed in Lambaréné, with a detailed mapping effort of schistosomiasis down to the individual level in the county.

The first cases of schistosomiasis were reported in the area in $1966 .{ }^{9}$ Only one MDA campaign for schistosomiasis has been conducted in the area for schistosomiasis control before the conduct of this study, and no other specific intervention measures were implemented. We, therefore, expected to find a high prevalence of schistosomiasis, and indeed, a very high prevalence of schistosomiasis is often found in the areas where access to PZQ treatment is scarce or unavailable. ${ }^{30,31}$ The moderate prevalence of schistosomiasis we found could suggest a stabilization of the prevalence of the disease in the area. An explanation for this may be the availability of the drug in the community. In addition to the scarce national campaigns of MDA, it should be noted that over the past 20 years, PZQ treatment has been provided free of charge to the infected population during the CERMEL routine or scheduled activities, being very active in the field of schistosomiasis, with significant impact in the community. Indeed, it has been demonstrated that treatment of the population with even a single dose of $\mathrm{PZQ}$ per year can be sufficient to reduce the morbidity of schistosomiasis in an endemic area. ${ }^{32,33}$ 
TABLE 3

Assessment of risk factors of schistosomiasis among the 614 study participants, April to July 2016, Lambaréné, Gabon

\begin{tabular}{|c|c|c|c|c|c|}
\hline & & & variate a & & \\
\hline & N & $n(\%)$ & $\mathrm{RR}$ & $95 \% \mathrm{Cl}(\mathrm{RR})$ & $P$-value \\
\hline Age (years) & & & & & 0.76 \\
\hline $5-9$ & 271 & $68(25.1)$ & 1 & - & \\
\hline $10-14$ & 307 & $83(27.0)$ & 1.08 & $0.82-1.42$ & \\
\hline $15-19$ & 36 & $8(23.2)$ & 0.88 & $0.46-1.69$ & \\
\hline Gender & & & & & 0.50 \\
\hline Female & 300 & $74(24.6)$ & 1 & - & \\
\hline Male & 314 & $85(27.0)$ & 1.06 & $0.89-1.26$ & \\
\hline Stream contact declared & & & & & $<0.001$ \\
\hline No & 344 & $48(16.9)$ & 1 & - & \\
\hline Yes & 266 & $110(48.7)$ & 2.96 & $2.20-4.00$ & \\
\hline Level of education & & & & & 0.43 \\
\hline First year & 127 & $40(36.5)$ & 1 & - & \\
\hline Second year & 116 & $31(26.7)$ & 0.85 & $0.57-1.26$ & \\
\hline Third year & 118 & $28(23.7)$ & 0.75 & $0.50-1.14$ & \\
\hline Fourth year & 133 & 35 (26.3) & 0.84 & $0.57-1.22$ & \\
\hline Fifth year & 120 & $25(20.3)$ & 0.66 & $0.43-1.02$ & \\
\hline
\end{tabular}

Another objective of the present study was to describe risk factors and factors associated with schistosomiasis. As an associated factor, we have assessed the relation of the history of PZQ taken with schistosomiasis, and we found that $10 \%$ of the study population declared to have taken PZQ in the past six months, regardless of the source of the drug. Surprisingly, more infected than uninfected children with schistosomiasis declared to have received treatment before, raising the issue of $P Z Q$ efficacy or reinfection. Indeed, in our study area, it is common to give $40 \mathrm{mg}$ of $P Z Q$ per body weight once to treat schistosomiasis as recommended by the $\mathrm{WHO},{ }^{34}$ although this protocol is sometimes found to have a limited efficacy. ${ }^{35}$ On the other hand, reinfection is common in endemic areas, ${ }^{35,36}$ assuming that those found infected despite the history of $P Z Q$ taken in the last months are reinfection cases. In both cases, there is a need for a proper investigation.

Assessing contacts of the study population with freshwater bodies considered as potential Schistosoma foci, nearly half of the population (44\%) declared to have had contact with open freshwater, implying that an important part of the

TABLE 4

Factors associated with schistosomiasis among the 614 study participants, April to July 2016, Lambaréné, Gabon

\begin{tabular}{|c|c|c|c|c|c|}
\hline & & & variate ar & & \\
\hline & N & $n(\%)$ & $\mathrm{RR}$ & $95 \% \mathrm{Cl}(\mathrm{RR})$ & $P$-value \\
\hline Hematuria & & & & & $<0.001$ \\
\hline No & 484 & 64 (13.2) & 1 & - & \\
\hline Yes & 130 & $95(73.1)$ & 5.53 & $4.30-7.10$ & \\
\hline Proteinuria & & & & & $<0.001$ \\
\hline No & 494 & $105(21.2)$ & 1 & - & \\
\hline Yes & 120 & $54(45.0)$ & 2.12 & $1.63-2.75$ & \\
\hline Hemoglobin rate* & & & & & 0.003 \\
\hline$\geq 11$ & 462 & $106(22.9)$ & 1 & - & \\
\hline$<11$ & 150 & $53(35.3)$ & 1.54 & $1.17-2.02$ & \\
\hline STH† & & & & & \\
\hline Trichuris trichiura & 49 & $23(46.9)$ & 1.82 & $1.30-2.56$ & 0.003 \\
\hline Hookworm & 18 & $4(22.2)$ & 0.79 & $0.33-1.89$ & 0.77 \\
\hline Ascaris lumbricoides & 15 & 8 (53.3) & 1.97 & $1.20-3.23$ & $0.04 \|$ \\
\hline Strongyloides stercoralis & 6 & 2 (33.3) & 1.19 & $0.38-3.74$ & $0.67 \|$ \\
\hline Any STH & 71 & $28(39.4)$ & 1.52 & $1.09-2.12$ & 0.03 \\
\hline Nutritional status & & & & & 0.61 \\
\hline Underweight & 33 & $6(18.2)$ & 0.68 & $0.32-1.42$ & \\
\hline Normal & 493 & $132(26.8)$ & 1 & - & \\
\hline Overweight & 57 & $15(26.3)$ & 0.98 & $0.62-1.55$ & \\
\hline Obese & 26 & 5 (19.2) & 0.72 & $0.32-1.60$ & \\
\hline History of praziquantel treatment $\ddagger$ & & & & & 0.007 \\
\hline No & 545 & $132(24.2)$ & 1 & - & \\
\hline Yes & 62 & $25(40.3)$ & 1.66 & $1.18-2.33$ & \\
\hline History of STH infection treatment§ & & & & & 0.15 \\
\hline No & 472 & $129(27.3)$ & 1 & - & \\
\hline Yes & 137 & $29(21.2)$ & 0.78 & $0.54-1.10$ & \\
\hline
\end{tabular}

STH = soil-transmitted helminth

*Two missing data.

† One hundred forty-two missing data.

$\ddagger$ Seven missing data.

§Five missing data.

\| Fisher's exact test. 
population is at risk of contact with Schistosoma parasites. When taking into account schistosomiasis status, the results demonstrate an ambiguous relationship leading to a couple of hypotheses. First, among participants found infected with schistosomiasis, no freshwater contact was declared for one of three of them. Subject to the reliability of their declarations, this fact could raise the possibility of the existence of additional, temporary schistosomiasis foci. In fact, Lambaréné is heavily irrigated by the tributaries of the Ogooué River. The rise of the river's waters during the rainy season is accompanied by flooding of the land, creating a significant number of temporary water pools, which can, thus, become potential foci of schistosomiasis, especially for school-age children. Second, among children not infected with S. haematobium, 35\% have declared to use stream water for household activities or bathing, suggesting either a low sensitivity of urine filtration technique for the diagnosis of schistosomiasis or a fact that all human-water contact points, particularly streams, are not schistosomiasis foci. Based on what has been described earlier, it appears that schistosomiasis foci are not only streams, and these foci are not homogeneous and geographically distributed in the study area as demonstrated by the geographical distribution map of our participants, which showed clusters of infected children (Figure 2B). Actually, the northwest of the town appears to be a high prevalence area for schistosomiasis, whereas the southeast appears to be a moderate prevalence area. Between the two, the disease is present, but at low prevalence. To better understand the situation of schistosomiasis in the area, the determinants of this picture need to be investigated.

Although freshwater contact is obviously the main risk factor for schistosomiasis, it is known that some factors such as age and gender can affect this risk factor through ageor gender-related behaviors. Some studies found that males are more at risk to be infected with schistosomiasis than females, ${ }^{37,38}$ whereas others have shown the contrary. ${ }^{39}$ For some authors, young age increases the risk to be infected with schistosomiasis. ${ }^{38,40}$ It has been suggested that the impact of culture on the population behavior with regard to contact with open freshwater could explain the difference observed and, therefore, affect the risk level of being infected. In the present study, neither gender nor age was associated with schistosomiasis. We, therefore, assume that the local culture does not lead to significant schistosomiasis exposure differences due to age- and gender-specific behaviors.

Hematuria is the main symptom known to be associated with urogenital schistosomiasis and very often used for indirect diagnosis of the disease. In this study, we found a strong association between any hematuria (microscopic and/or visible) and schistosomiasis. The situation was similar for proteinuria, meaning that these two biomarkers can be of interest for the diagnosis of schistosomiasis in our population. This question will be addressed in a further article. On the contrary to anemia, malnutrition was not associated with schistosomiasis. This corroborates the findings of Abdi et al. ${ }^{41}$ who also found no association between intestinal schistosomiasis and malnutrition status among schoolchildren living in northwestern Ethiopia. ${ }^{41}$ Even in a highly endemic area, Munisi and collaborators found no association between S. mansoni infection malnutrition among Tanzanian schoolchildren. ${ }^{42}$ However, our results opposed the findings of some other authors who reported that malnutrition is associated with
S. haematobium infection in children living in moderate or highly endemic areas for schistosomiasis. ${ }^{6,43}$ These conflicting results suggest the existence of other determinants, which could affect the association between the disease and nutritional status. However, this has to be investigated in more detail prospectively in our population. In the case of the present study, we hypothesize a good nutritional status of schoolchildren in Lambaréné.

This study confirms once more that Lambaréné is endemic for STH infections, particularly for $A$. lumbricoides, $T$. trichiura, hookworm, and $S$. stercoralis, ${ }^{12}$ with $T$. trichiura being the predominant STH species, followed by hookworm and A. lumbricoides. On the contrary to the vicinity of Lambaréné where a moderate prevalence of STH infections was recently reported, ${ }^{17}$ the low prevalence we report here could be explained by the availability of anthelminthic drug in the area, particularly albendazole, which is very often used for selfmedication by the local population. Indeed, about $25 \%$ of study participants have declared having received anti-STH treatment in the last six months preceding the study. Moreover, the morbidity of STH infections is associated with the intensity of the disease. Heaviest infection is indeed associated with anemia or malnutrition, among others. In our study population, all cases of STH infections were of light intensity, assuming their non-association with anemia and malnutrition. This result supports our finding on the association of anemia with schistosomiasis in our study population and consolidates the low number of underweight cases we reported.

The geographical distribution of children infected with STHs shows a homogeneous spread of these parasites across the study area (Figure $2 \mathrm{C}$ ) and, therefore, their local coexistence with schistosomiasis. Schistosomiasis-STH infection co-endemicity is commonly reported in sub-Saharan countries. ${ }^{44-46}$ Assessing the coinfection with both trematodes and intestinal nematodes, we found an increase in risk of having schistosomiasis when infected with STHs, particularly with $T$. trichiura and $A$. lumbricoides. Although some authors found no association between schistosomiasis and STH infections, such as Njaanake et al. ${ }^{45}$ in Kenyan schoolchildren from two villages, our results are in line with what was reported by Molvik et al. ${ }^{44}$ on South African schoolchildren living in moderate prevalence areas for schistosomiasis and STH infections. Even some immunological mechanism can explain that fact; we hypothesize that this association could be due to the fact that both types of infection share the same environmental risk factors. If the absence of an association with $S$. stercoralis could be due to a lack of statistical power (we found very few cases of strongyloidiasis), the absence of association with hookworm infection, which was more prevalent than ascariasis in our cohort, would support our hypothesis. Indeed, it is known that the life cycle of hookworm requires hot or sandy soils, in contrast to those of Schistosoma spp., Ascaris spp., and Trichuris spp. Trichuris trichiura eggs, for instance, must remain in warm moist soil to become infectious. In any case, these results show the necessity to take into account the interaction between schistosomiasis and STH infections, especially with trichuriasis and ascariasis for assessment of the effect of one of these diseases, in particular at the immunological level.

\section{CONCLUSION}

Lambaréné represents an area of moderate prevalence for schistosomiasis and low prevalence for STH infections. Our 
results emphasize the necessity to fully implement the recommendations of the $\mathrm{WHO}$ to improve the situation of schistosomiasis in the area where no additional risk factors other than human-water contact could be identified among schoolchildren. To that aim, hematuria and proteinuria strongly associated with the infection could help. Although the picture of schistosomiasis in Lambaréné becomes clearer, the need to establish the transmission dynamic remains. A pilot malacological study conducted in the area few years before already provides some helpful information.

Received November 7, 2019. Accepted for publication April 18, 2020.

Published online May 18, 2020.

Acknowledgments: We would like to thank the study team, notably fieldworkers (Prisca Noelle Bouyebi Bouchoko Eréné, Eliane Moussouami, Guy-Gaspard Nzadi, and Olivier Koumba), data entry clerks (Prisca Noelle Bouyebi Bouchoko Eréné and Eliane Moussouami), nurses (Ivy Marion Moussounda Mondjo, Lucie Prisca Bena, Marie Yvonne Essongue, and Annie Christelle Ndong), and the laboratory technicians' team for their special commitment during this study. We especially thank the schoolchildren for their voluntary participation in this study, their parents, teachers, and the administrative authorities of health and education for allowing us to carry out this study.

Financial support: We are grateful to CERMEL for the financial and logistic support for the study. J. C. D. A. was supported financially by Deutsche Forschungsgemeinschaft German-African Cooperation Projects in Infectiology GZ:MO1071/12-1 AOBJ:620617.

Disclosure: A. A. A. and M. G. P. are members of CANTAM (EDCTPRegNet2015-1045) and PANDORA-ID-Net (EDCTP Grant Agreement RIA2016E-1609) networks.

Authors' addresses: Jean Claude Dejon Agobé, Centre de Recherches Médicales de Lambaréné (CERMEL), Lambaréné, Gabon, and Division of Internal Medicine, Center of Tropical Medicine and Travel Medicine, Amsterdam University Medical Centers, location AMC, University of Amsterdam, Amsterdam, The Netherlands, E-mail: jcagobe@gmail.com. Yabo Josiane Honkpehedji and Jeannot Fréjus Zinsou, Centre de Recherches Médicales de Lambaréné (CERMEL), Lambaréné, Gabon, and Department of Parasitology, Leiden University Medical Center, Leiden, The Netherlands, E-mails: hyjosy@ gmail.com and zinaff@yahoo.fr. Jean Ronald Edoa, Bayodé Roméo Adegbite and Ance Mangaboula, Centre de Recherches Médicales de Lambaréné (CERMEL), Lambaréné, Gabon, E-mails: jronaldedoa@ gmail.com, aromakobs@gmail.com, and ance.mangaboula@ cermel.org. Selidji Todagbe Agnandji, Centre de Recherches Médicales de Lambaréné (CERMEL), Lambaréné, Gabon, and Institut für Tropenmedizin, Eberhad Karls Universität Tübingen, Tübingen, Germany, E-mail: agnandjis@cermel.org. Ghyslain Mombo-Ngoma, Centre de Recherches Médicales de Lambaréné (CERMEL), Lambaréné, Gabon, Institut für Tropenmedizin, Eberhad Karls Universität Tübingen, Germany, and I. Department of Medicine, Bernhard Nocht Institute for Tropical Medicine, University Medical Centre-Hamburg-Eppendorf, Hamburg, Germany, E-mail: ghyslain.mombongoma@cermel.org. Michael Ramharter, I. Department of Medicine, Bernhard Nocht Institute for Tropical Medicine, University Medical Centre-Hamburg-Eppendorf, Hamburg, Germany, E-mail: ramharter@bnitm.de. Bertrand Lell, Centre de Recherches Médicales de Lambaréné (CERMEL), Lambaréné, Gabon, Division of Infectious Diseases and Tropical Medicine, Department of Medicine, Medical University of Vienna, Vienna, Austria, Institut für Tropenmedizin, Eberhad Karls Universität Tübingen, and German Center for Infection Research, Tübingen, Germany, E-mail: bertrand.lell@ gmail.com. Peter Gottfried Kremsner, Centre de Recherches Médicales de Lambaréné (CERMEL) and German Center for Infection Research (DZIF), African partner institution, CERMEL, Lambaréné, Gabon; Institut für Tropenmedizin, Eberhad Karls Universität Tübingen, and German Center for Infection Research (DZIF), Tübingen, Germany, E-mail: peter.kremsner@uni-tuebingen.de. Martin Peter Grobusch, Centre de Recherches Médicales de Lambaréné (CERMEL), Lambaréné, Gabon, Division of Internal Medicine, Center of Tropical Medicine and Travel Medicine, Amsterdam University Medical Centers, location AMC, University of Amsterdam, Amsterdam, The Netherlands, and Institut für
Tropenmedizin, Eberhad Karls Universität Tübingen, Tübingen, Germany, E-mail: m.p.grobusch@amsterdamumc.nl. Ayola Akim Adegnika, Centre de Recherches Médicales de Lambaréné (CERMEL), Lambaréné, Gabon, Department of Parasitology, Leiden University Medical Center, Leiden, The Netherlands, Institut für Tropenmedizin, Eberhad Karls Universität Tübingen, Tübingen, Germany, German Center for Infection Research (DZIF), African Partner Institution, CERMEL, Lambaréné, Gabon, and German Center for Infection Research (DZIF), Partner Site, Tübingen, Germany, E-mail: aadegnika@cermel.org.

\section{REFERENCES}

1. CDC, 2019. Schistosomiasis. Available at: https://www.cdc.gov/ parasites/schistosomiasis/index.html. Accessed July 28, 2019.

2. World Health Organization EMRO, 2019. Schistosomiasis-Health Topics. Available at: http://www.emro.who.int/health-topics/ schistosomiasis/. Accessed September 1, 2019.

3. World Health Organization, 2019. Soil-Transmitted Helminth Infections. Available at: https://www.who.int/news-room/factsheets/detail/soil-transmitted-helminth-infections. Accessed July 28, 2019.

4. World Health Organization, 2018. Soil-Transmitted Helminthiases: Number of Children (Pre-SAC and SAC) Requiring Preventive Chemotherapy for Soil-Transmitted Helminthiases. Available at: http://apps.who.int/neglected_diseases/ntddata/ sth/sth.html. Accessed March 19, 2020.

5. Hotez PJ, Kamath A, 2009. Neglected tropical diseases in subSaharan Africa: review of their prevalence, distribution, and disease burden. PLoS Negl Trop Dis 3: e412.

6. Frigerio S, Macario M, lacovone G, Dussey-Comlavi KJ, Narcisi P, Ndiaye AT, Moramarco S, Alvaro R, Palombi L, Buonomo E, 2016. Schistosoma haematobium infection, health and nutritional status in school-age children in a rural setting in northern Senegal. Minerva Pediatr 68: 282-287.

7. World Health Organization, 2017. Fact Sheet; Schistosomiasis. Available at: http://www.who.int/mediacentre/factsheets/ fs115/en/. Accessed July 6, 2017.

8. World Health Organization, 2006. Preventive Chemotherapy in Human Helminthiasis : Coordinated Use of Anthelminthic Drugs in Control Interventions : A Manual for Health Professionals and Programme Managers. Geneva, Switzerland: World Health Organization. Available at: http://www.who.int/iris/handle/ 10665/43545. Accessed July 13, 2017.

9. Deschiens R, Poirier A, 1967. Epidemiologic and clinical aspects of Schistosoma intercalatum bilharziasis in Gabon. Bull Soc Pathol Exot Filiales 60: 228-240.

10. CNRS-World Health Organization, 1987. Gabon. In: Atlas of the Global Distribution of Schistosomiasis. Bordeaux, France: Presses Universitaires de Bordeaux, 157-160.

11. Mintsa Nguema $R$ et al., 2018. Baseline mapping of schistosomiasis and soil transmitted helminthiasis in the northern and eastern health regions of Gabon, central Africa: recommendations for preventive chemotherapy. Trop Med Infect Dis 3: 119

12. Adegnika AA, Ramharter M, Agnandji ST, Ateba Ngoa U, Issifou S, Yazdanbakhsh M, Kremsner PG, 2010. Epidemiology of parasitic co-infections during pregnancy in Lambaréné, Gabon. Trop Med Int Health 15: 1204-1209.

13. Mombo-Ngoma G et al., 2017. Urogenital schistosomiasis during pregnancy is associated with low birth weight delivery: analysis of a prospective cohort of pregnant women and their offspring in Gabon. Int J Parasitol 47: 69-74.

14. Ateba-Ngoa U, Jones S, Zinsou JF, Kassa Kassa RF, Honkpehedji J, Dejon Agobé JC, Massinga-Loembe M, Mordmüller B, Bousema T, Yazdanbakhsh M, 2016. Associations between helminth infections, Plasmodium falciparum parasite carriage and antibody responses to sexual and asexual stage malarial antigens. Am J Trop Med Hyg 95: 394-400.

15. Gabon - Fiche pays - PopulationData.net, 2017. PopulationData.net. Available at: $h$ ttps://www.populationdata.net/pays/gabon/. Accessed December 31, 2017.

16. PLMP, 2015. Enquêtes Épidémiologiques sur la Schistosomose, les Géohelminthiases et les Filarioses. Libreville, Gabon: 
Ministère de la Santé, de la Prévoyance Soiale et de la Solidarité Nationale du Gabon.

17. Dejon-Agobé JC et al., 2018. Schistosoma haematobium effects on Plasmodium falciparum infection modified by soil-transmitted helminths in school-age children living in rural areas of Gabon. PLoS Negl Trop Dis 12: e0006663.

18. Daniel WW, 1999. Biostatistics: A Foundation for Analysis in the Health Sciences, 7th edition. New York, NY: John Wiley \& Sons.

19. Dejon-Agobé JC, 2019. Schistosoma haematobium infection morbidity, praziquantel effectiveness and reinfection rate among children and young adults in Gabon. Parasit Vectors 12: 577.

20. Katz N, Chaves A, Pellegrino J, 1972. A simple device for quantitative stool thick-smear technique in Schistosomiasis mansoni. Rev Inst Med Trop Sao Paulo 14: 397-400.

21. Harris PA, Taylor R, Thielke R, Payne J, Gonzalez N, Conde JG, 2009. Research electronic data capture (REDCap)-a metadatadriven methodology and workflow process for providing translational research informatics support. J Biomed Inform 42: 377-381.

22. World Health Organization, 2011. Haemoglobin Concentrations for the Diagnosis of Anaemia and Assessment of Severity. Available at: http://www.who.int/vmnis/indicators/haemoglobin.pdf. Accessed March 15, 2018.

23. World Health Organization, 2007. Growth Reference Data for 5-19 Years. Available at: http://www.who.int/growthref/en/. Accessed June 26, 2017.

24. World Health Organization, 2004. Team WHOSD and M for PD and VC, UNICEF. Prevention and Control of Schistosomiasis and Soil-Transmitted Helminthiasis. Available at: http:// www.who.int/iris/handle/10665/68607. Accessed June 25, 2017.

25. ICH, 2019. Official Web Site: ICH. Available at: https:// www.ich.org/home.html. Accessed April 24, 2019.

26. WMA, 2018. The World Medical Association-Déclaration d'Helsinki de L'AMM - Principes éthiques applicables à la recherche médicale impliquant des êtres humains. Available at: https:// www.wma.net/fr/policies-post/declaration-dhelsinki-de-lammprincipes-ethiques-applicables-a-la-recherche-medicale-impliquantdes-etres-humains/. Accessed April 23, 2018.

27. Labuda LA, de Jong SE, Meurs L, Amoah AS, Mbow M, AtebaNgoa U, van der Ham AJ, Knulst AC, Yazdanbakhsh M, Adegnika AA, 2014. Differences in innate cytokine responses between European and African children. PLoS One 9: e95241.

28. Cunin P, Tchuem Tchuenté LA, Poste B, Djibrilla K, Martin PMV, 2003. Interactions between Schistosoma haematobium and Schistosoma mansoni in humans in north Cameroon. Trop Med Int Health 8: 1110-1117.

29. Almeda J, Ascaso C, Marçal GA, Corachan M, Southgate VR, Rollinson D, 1996. Morphometric variability of Schistosoma intercalatum eggs: a diagnostic dilemma. J Helminthol 70: 97-102.

30. Morenikeji O, Quazim J, Omoregie C, Hassan A, Nwuba R, Anumudu C, Adejuwon S, Salawu O, Jegede A, Odaibo A, 2014 A cross-sectional study on urogenital schistosomiasis in children; haematuria and proteinuria as diagnostic indicators in an endemic rural area of Nigeria. Afr Health Sci 14: 390-396.

31. Bocanegra $C$ et al., 2015. Epidemiology of schistosomiasis and usefulness of indirect diagnostic tests in school-age children in Cubal, central Angola. PLoS Negl Trop Dis 9: e0004055.

32. Koukounari A, Gabrielli AF, Touré S, Bosqué-Olivia E, Zhang Y, Sellin B, Donnelly CA, Fenwick A, Webster JP, 2007.
Schistosoma haematobium infection and morbidity before and after large-scale administration of praziquantel in Burkina Faso. $J$ Infect Dis 196: 659-669.

33. Tohon ZB, Mainassara HB, Garba A, Mahamane AE, BosquéOlivia E, Ibrahim ML, Duchemin JB, Chanteau S, Boisier P, 2008. Controlling schistosomiasis: significant decrease of anaemia prevalence one year after a single dose of praziquantel in Nigerien schoolchildren. PLoS Negl Trop Dis 2: e241.

34. OMS, 1997. Fiches Modèles OMS d'Information à l'Usage des Prescripteurs: Médicaments Utilisés en Parasitologie Deuxième Edition: Helminthes: Schistosomiase: Praziquantel. Available at: http://apps.who.int/medicinedocs/en/d/Jh2923f/ 3.7.1.html. Accessed February 19, 2018.

35. Kabuyaya M, Chimbari MJ, Manyangadze T, Mukaratirwa S, 2017. Efficacy of praziquantel on Schistosoma haematobium and re-infection rates among school-going children in the Ndumo area of uMkhanyakude district, KwaZulu-Natal, South Africa. Infect Dis Poverty 6: 83.

36. Satayathum SA, Muchiri EM, Ouma JH, Whalen $\mathrm{CC}$, King $\mathrm{CH}$, 2006. Factors affecting infection or reinfection with Schistosoma haematobium in coastal Kenya: survival analysis during a nine-year, school-based treatment program. Am J Trop Med Hyg 75: 83-92.

37. Zongo D, Kabre BG, Dayeri D, Savadogo B, Poda JN, 2012. Étude comparative de la transmission de la schistosomiase (formes urinaire et intestinale) dans dix sites du Burkina Faso. Méd Santé Trop 22: 323-329.

38. Hammam HM et al., 2000. The epidemiology of schistosomiasis in Egypt: Qena governorate. Am J Trop Med Hyg 62: 80-87.

39. Nkegbe E, 2010. Sex prevalence of schistosomiasis among school children in five communities in the lower River Volta Basin of south eastern Ghana. Afr J Biomed Res 13: 87-88.

40. Zida A et al., 2016. Epidemiological and clinical aspects of urogenital schistosomiasis in women, in Burkina Faso, west Africa. Infect Dis Poverty 5: 81.

41. Abdi M, Nibret E, Munshea A, 2017. Prevalence of intestinal helminthic infections and malnutrition among schoolchildren of the Zegie Peninsula, northwestern Ethiopia. J Infect Public Health 10: 84-92.

42. Munisi DZ, Buza J, Mpolya EA, Kinung'hi SM, 2016. Schistosoma mansoni infections, undernutrition and anaemia among primary schoolchildren in two onshore villages in Rorya District, northwestern Tanzania. PLoS One 11: e0167122.

43. Botelho MC, Machado A, Carvalho A, Vilaça M, Conceicão O, Rosa F, Alves H, Richter J, Bordalo AA, 2016. Schistosoma haematobium in Guinea-Bissau: unacknowledged morbidity due to a particularly neglected parasite in a particularly neglected country. Parasitol Res 115: 1567-1572.

44. Molvik M, Heiland E, Zulu SG, Kleppa E, Lillebo K, Gundersen SG, Kvalsvig JD, Taylor M, Kjetland EF, Vennervald BJ, 2017. Coinfection with Schistosoma haematobium and soil-transmitted helminths in rural South Africa. South Afr J Sci 113: 1-6.

45. Njaanake $K H$, Vennervald $B J$, Simonsen PE, Simonsen PE, Madsen H, Mukoko DA, Kimani G, Jaoko WG, Estambale BB, 2016. Schistosoma haematobium and soil-transmitted helminths in Tana delta district of Kenya: infection and morbidity patterns in primary schoolchildren from two isolated villages. BMC Infect Dis 16: 57.

46. Boko PM, Ibikounle M, Onzo-Aboki A, Tougoue JJ, Sissino Y, Batcho W, Kinde-Gazard D, Kabore A, 2016. Schistosomiasis and soil transmitted helminths distribution in Benin: a baseline prevalence survey in 30 districts. PLoS One 11: e0162798. 